\title{
The number of rooted trees of given depth
}

\author{
Péter Pál Pach \\ Department of Computer Science and Information Theory \\ University of Technology and Economics \\ Budapest, Hungary \\ ppp24@cs.elte.hu \\ Gabriella Pluhár \\ Department of Algebra and Number Theory, \\ Eötvös Loránd University \\ Budapest, Hungary \\ plugab@cs.elte.hu \\ András Pongrácz \\ Department of Mathematics and its Applications, \\ Central European University \\ Budapest, Hungary \\ andras.pongracz@lix.polytechnique.fr \\ Csaba Szabó \\ Department of Algebra and Number Theory, \\ Eötvös Loránd University \\ Budapest, Hungary \\ csaba@cs.elte.hu
}

Submitted: Feb 26, 2011; Accepted: May 16, 2013; Published: May 24, 2013

Mathematics Subject Classifications: 05C30, 05A15, 05A16

\begin{abstract}
In this paper it is shown that the logarithm of the number of non-isomorphic rooted trees of depth $k \geqslant 3$ with $n$ vertices is asymptotically $\frac{\pi^{2}}{6} \cdot \frac{n}{\log \log \ldots \log n}$, where $\log$ is iterated $k-2$ times in the denominator.
\end{abstract}

Keywords: tree, depth, counting 


\section{Introduction}

In 1889 Cayley showed that there are $n^{n-2}$ labelled trees on $n$ vertices. In 1948 asymptotic formulas were given for the number of unlabelled trees and unlabelled rooted trees. In the seminal paper of Otter [5] it is shown that the number of unlabelled trees of order $n$ is asymptotically $b n^{-5 / 2} \alpha^{n}(1+O(1 / n))$, and the number of unlabelled rooted trees of order $n$ is asymptotically $c n^{-3 / 2} \alpha^{n}(1+O(1 / n))$, where $\alpha=2.95576 \ldots, b=0.5349 \ldots$ and $c=0.4399 \ldots$ All results about counting trees are summarized in the book of Drmota [2]. Several parameters of trees were analyzed in detail, for example, the average depth, the distribution of the depth in unlabelled rooted trees [3] and random $d$-ary trees, etc. For the distribution of the depth of binary unlabelled rooted trees see [1].

In this paper we count the number of rooted trees of given depth on $n$ vertices. We show that the logarithm of the number of rooted trees of depth $k \geqslant 3$ is asymptotically $\frac{\pi^{2}}{6} \cdot \frac{n}{\log \log \ldots \log n}$, where $\log$ is iterated $k-2$ times in the denominator.

\section{Generating functions}

Denote by $f_{k}(n)$ the number of $n$-vertex rooted trees of depth at most $k$. A rooted tree of depth 0 is a single point. A rooted tree of depth 1 has a root and $n-1$ leaves all connected to the root. Hence $f_{1}(n)=1$ for all $n \geqslant 1$. The 5 -vertex trees of depth at most 2 are shown on Figure 1 . Thus $f_{2}(5)=5$. It is easy to find a general formula for the number of rooted trees of depth at most 2 .

Lemma 2.1. $f_{2}(n)=p(n-1)$, where $p(m)$ denotes the number of partitions of $m$.

Proof. Let us omit the root of an $n$-vertex tree of depth at most 2 . Then we obtain some (rooted) trees of depth at most 1 with altogether $n-1$ vertices. Trees of depth at most 1 are uniquely determined by the number of their vertices. Hence, we have exactly as many such configurations as many partitions of $n-1$. Thus $f_{2}(n)=p(n-1)$.

For a fixed $k$ let $F_{k}(x)$ denote the generating function of the sequence $f_{k}(n)$.

$$
F_{k}(x)=\sum_{n=1}^{\infty} f_{k}(n) x^{n}
$$

By Lemma 2.1, $F_{2}(x)=\sum_{n=1}^{\infty} p(n-1) x^{n}=x P(x)$, where $P(x)$ denotes the generating function of the partitions of $n$. By the Hardy-Ramanujan formula $f_{2}(n) \sim \frac{1}{4 n \sqrt{3}} e^{\pi \sqrt{\frac{2 n}{3}}}$, which shows the asymptotic behaviour of $f_{2}(n)$. For more details see [6]. To attain a recurrence formula for $F_{k}(x)$, we use again the idea of chopping the tree: Omit the root of an $n$-vertex tree of depth at most $k$. The remaining part of the graph is a forest consisting of trees of depth at most $k-1$ with $n-1$ vertices altogether. Let $\mu_{j}$ be the number of 

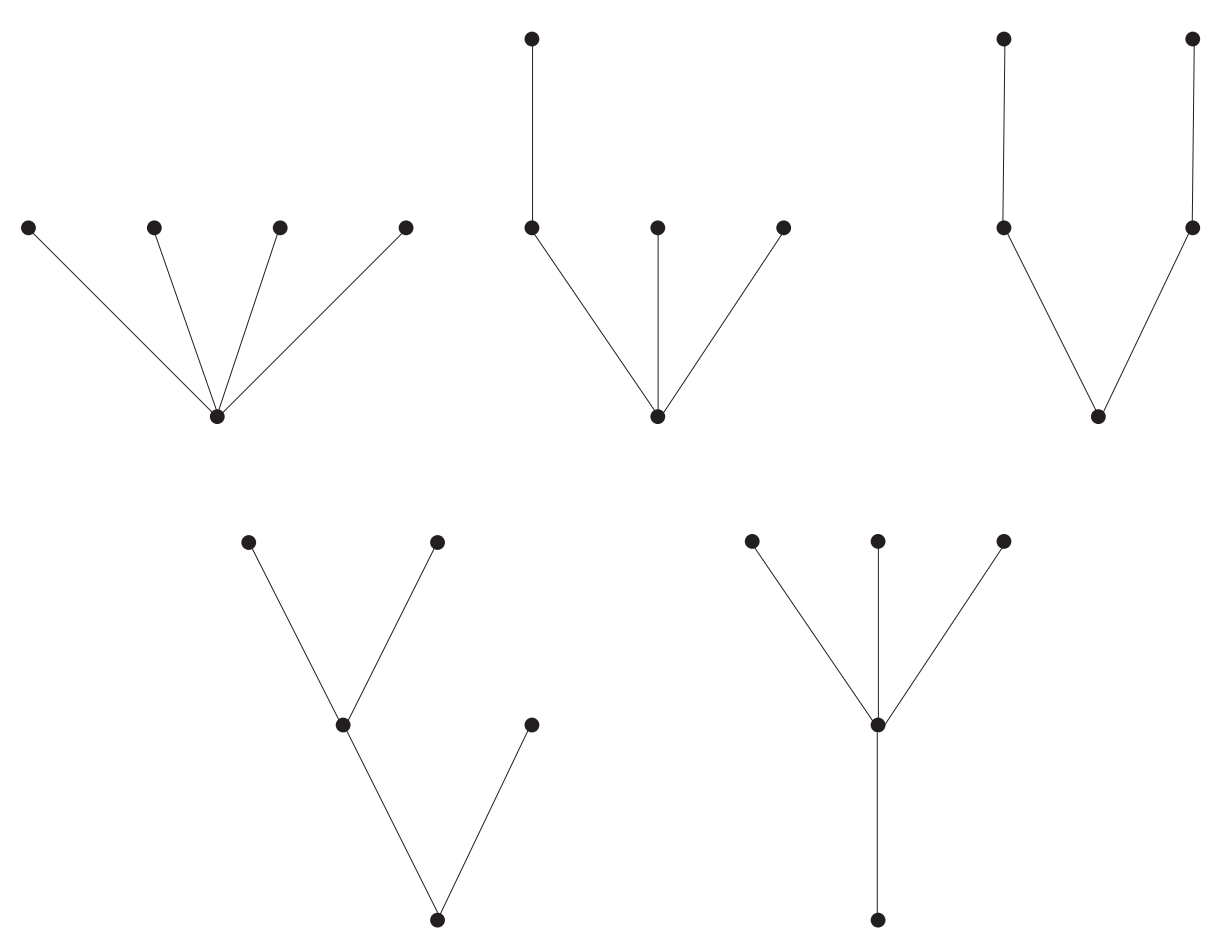

Figure 1:

rooted trees with $j$ vertices after the chopping. There are $\left(\begin{array}{c}f_{k-1}(j)+\mu_{j}-1 \\ \mu_{j}\end{array}\right)$ ways to choose $\mu_{j}$ trees with $j$ vertices. Thus we have the following recurrence formula

$$
f_{k}(n)=\sum_{\sum i \mu_{i}=n-1}\left(\prod_{j=1}^{n-1}\left(\begin{array}{c}
f_{k-1}(j)+\mu_{j}-1 \\
\mu_{j}
\end{array}\right)\right)
$$

This technique, and the following formulas can be found in [4], but we summarize the proofs for the reader's convenience.

Theorem 2.2. Let $k \geqslant 2$. Then the generating function of the sequence $f_{k}(n)$ is

$$
F_{k}(x)=x \prod_{j=1}^{\infty}\left(1-x^{j}\right)^{-f_{k-1}(j)}
$$

and satisfies the recurrence formulas

$$
\begin{gathered}
F_{k}(x)=x \exp \left(\sum_{m=1}^{\infty} \frac{1}{m} \sum_{j=1}^{\infty} f_{k-1}(j) x^{j m}\right) \\
F_{k}(x)=x \exp \left(\sum_{m=1}^{\infty} \frac{1}{m} F_{k-1}\left(x^{m}\right)\right)
\end{gathered}
$$


Proof. According to the generalized binomial theorem, for every $|x|<1$ we have

$$
\left(1-x^{j}\right)^{-f_{k-1}(j)}=\sum_{\mu_{j}=0}^{\infty}\left(\begin{array}{c}
-f_{k-1}(j) \\
\mu_{j}
\end{array}\right) \cdot\left(-x^{j}\right)^{\mu_{j}}=\sum_{\mu_{j}=0}^{\infty}\left(\begin{array}{c}
f_{k-1}(j)+\mu_{j}-1 \\
\mu_{j}
\end{array}\right) x^{j \mu_{j}}
$$

Thus the coefficient of $x^{n-1}$ in the expression $\prod_{j=1}^{\infty}\left(1-x^{j}\right)^{-f_{k-1}(j)}$ is

$$
\sum_{\mu_{1}+2 \mu_{2}+\cdots+(n-1) \mu_{n-1}=n-1}\left(\prod_{j=1}^{n-1}\left(\begin{array}{c}
f_{k-1}(j)+\mu_{j}-1 \\
\mu_{j}
\end{array}\right)\right)
$$

and this is exactly $f_{k}(n)$ by (1). By expanding the Taylor-series of $\log \left(1-x^{j}\right)$, for $0<x<1$ we obtain

$$
\begin{aligned}
\log F_{k}(x) & =\log x+\sum_{j=1}^{\infty}\left(-f_{k-1}(j)\right) \log \left(1-x^{j}\right) \\
& =\log x+\sum_{j=1}^{\infty}\left(-f_{k-1}(j)\right) \sum_{m=1}^{\infty}\left(-\frac{1}{m} x^{j m}\right) \\
& =\log x+\sum_{m=1}^{\infty} \frac{1}{m} \sum_{j=1}^{\infty} f_{k-1}(j) x^{j m}=\log x+\sum_{m=1}^{\infty} \frac{1}{m} F_{k-1}\left(x^{m}\right)
\end{aligned}
$$

which is equivalent to $(\mathrm{F} 1)$.

\section{Preliminary calculations}

We give a list of elementary analytic calculations often used in the estimations of the generating functions. Those not interested in the technical details of these easy calculations can skip the whole section.

Definition 3.1. For $m \geqslant 1$ we denote by $L_{m}(x)$ the $m$-th iterated logarithm function $\log \log \ldots \log x$. Similarly, $E_{m}(x)$ denotes the $m$-th iterated exponential function $\exp \exp \ldots \exp x$.

Lemma 3.2. The following rules apply for the functions $L_{m}$ and $E_{m}$.

(i) For $x, y \geqslant 2$ we have $\log x \leqslant \log (x+y) \leqslant \log x+\log y$.

(ii) For every $m \geqslant 2$ and every large enough $x, y$ we have $L_{m}(x) \leqslant L_{m}(x y) \leqslant L_{m}(x)+$ $L_{m}(y)$.

(iii) $E_{m}(x / 2) \leqslant E_{m}(x)^{1 / 2}$ and $E_{m}(x / 3) \leqslant E_{m}(x)^{1 / 3}$ for large enough $x$.

(iv) For all $C_{1}, C_{2}>0$ there exits a constant $C_{3}>0$ with $C_{1} E_{m}\left(x+C_{2}\right) \leqslant E_{m}\left(x+C_{3}\right)$ for large enough $x$. 
Proof. As $\log x$ is increasing we have $\log x \leqslant \log (x+y)$ for $x, y \geqslant 2$. For the other inequality without loss of generality assume that $x \leqslant y$. Then $\log (x+y)=\log \left(y\left(1+\frac{x}{y}\right)\right)=$ $\log y+\log \left(1+\frac{x}{y}\right) \leqslant \log y+\log 2 \leqslant \log y+\log x$.

Let $x, y$ be large enough such that $L_{m}(x) \geqslant 2$ and $L_{m}(y) \geqslant 2$. Then according to the monotonicity of $L_{m}(x)$ we have $L_{m}(x) \leqslant L_{m}(x y)$. Applying $(i)$ repeatedly, we obtain $L_{m}(x y) \leqslant L_{m-1}\left(L_{1}(x)+L_{1}(y)\right) \leqslant L_{m-2}\left(L_{2}(x)+L_{2}(y)\right) \leqslant \cdots \leqslant L_{m}(x)+L_{m}(y)$.

Item (iii) is shown by induction. It is clear for $m=1$. For $m>1$ we have $E_{m}(u / 2)=\exp \left(E_{m-1}(u / 2)\right) \leqslant \exp \left(E_{m-1}(u)^{1 / 2}\right) \leqslant E_{m}(u)^{1 / 2}$ by the induction hypothesis, if $E_{m-1}(u) \geqslant 4$.

Item (iv) follows from the formula $E_{m}(x+y) \geqslant E_{m}(x) E_{m}(y)$ for large enough $x, y$.

Throughout the paper we estimate certain power series coefficientwise. That is, $\leqslant$ coeff is a partial order on the set of real power series, and $\sum_{n=0}^{\infty} a_{n} x^{n} \leqslant$ coeff $\sum_{n=0}^{\infty} b_{n} x^{n}$ if and only if $a_{n} \leqslant b_{n}$ for all $n \geqslant 0$. The following rules are going to be used several times.

Lemma 3.3. Let $\sum_{n=0}^{\infty} a_{n} x^{n}$ and $\sum_{n=0}^{\infty} b_{n} x^{n}$ be two (formal) power series. Then

(i) $\exp \left(\sum_{n=0}^{\infty} a_{n} x^{n}\right)=\sum_{n=0}^{\infty}\left(\sum_{i=0}^{\infty} \frac{1}{i !} \sum_{k_{1}+\cdots+k_{i}=n} a_{k_{1}} \cdots a_{k_{i}}\right) x^{n}$,

(ii) if $0 \leqslant$ coeff $\sum_{n=0}^{\infty} a_{n} x^{n} \leqslant$ coeff $\sum_{n=0}^{\infty} b_{n} x^{n}$, then

$$
\exp \left(\sum_{n=0}^{\infty} a_{n} x^{n}\right) \leqslant \text { coeff } \exp \left(\sum_{n=0}^{\infty} b_{n} x^{n}\right)
$$

Proof. The first item follows from $\exp (y)=\sum_{i=0}^{\infty} \frac{1}{i !} y^{i}$, and item $(i i)$ is a direct consequence of $(i)$.

\section{Asymptotic formulas}

In this section, we prove the main theorem of the paper.

Theorem 4.1. The sequences $f_{k}(n)$ satisfy the following asymptotic formulas

(1) $f_{2}(n)=p(n-1) \sim \frac{1}{4 n \sqrt{3}} e^{\pi \sqrt{\frac{2 n}{3}}}$

(2) $\log f_{k}(n)=\frac{\pi^{2}}{6} \cdot \frac{n}{L_{k-2}(n)} \cdot\left(1+O_{k}\left(\frac{L_{k-1}(n)}{L_{k-2}(n)}\right)\right)$ for $k>2$,

where $L_{m}(x)$ denotes the $m$-th iterated logarithm function $\log \log \cdots \log x$.

The first statement of this theorem is a direct consequence of Lemma 2.1. For the second item a series of lemmas is needed. 


\subsection{The lower estimation}

First we give a lower bound for $k=3$.

Lemma 4.2. $\log f_{3}(n) \geqslant \frac{\pi^{2}}{6} \cdot \frac{n}{\log n}+O\left(n \frac{\log \log n}{\log ^{2} n}\right)$

Proof. According to formula (F1) we have the trivial lower bound

$$
F_{3}(x) \geqslant_{\text {coeff }} x \exp \left(\sum_{n=1}^{\infty} f_{2}(n) x^{n}\right)
$$

By expanding the exponential function for $f_{3}(n)$ we obtain

$$
f_{3}(n) \geqslant \sum_{i=1}^{n} \frac{1}{i !} \sum_{a_{1}+\cdots+a_{i}=n-1} f_{2}\left(a_{1}\right) \cdots f_{2}\left(a_{i}\right)
$$

Consider a term where $i=\frac{\pi^{2}}{6} \cdot \frac{n}{\log ^{2} n} \cdot\left(1+O\left(\frac{\log \log n}{\log n}\right)\right)$ and $a_{1}=\cdots=a_{i}=\frac{n-1}{i}$. Then $a_{1}=\cdots=a_{i}=\frac{6}{\pi^{2}} \cdot \log ^{2} n \cdot\left(1+O\left(\frac{\log \log n}{\log n}\right)\right) \cdot\left(1-\frac{1}{n}\right)=\frac{6}{\pi^{2}} \cdot \log ^{2} n \cdot\left(1+O\left(\frac{\log \log n}{\log n}\right)\right)$.

By estimating $\log i$ ! with Stirling's formula and by using that for large enough $m$ the inequality $f_{2}(m-1) \geqslant \exp \left(\pi \sqrt{\frac{2 m}{3}}-2 \log m\right)$ holds, we obtain

$$
\begin{aligned}
& \log f_{3}(n) \geqslant-i \log i+i \log f_{2}\left(a_{1}\right) \\
& \geqslant-\frac{\pi^{2}}{6} \cdot \frac{n}{\log ^{2} n} \cdot\left(1+O\left(\frac{\log \log n}{\log n}\right)\right) \cdot \log \left(\frac{\pi^{2}}{6} \cdot \frac{n}{\log ^{2} n} \cdot\left(1+O\left(\frac{\log \log n}{\log n}\right)\right)\right) \\
& \quad+\frac{\pi^{2}}{6} \cdot \frac{n}{\log ^{2} n} \cdot\left(1+O\left(\frac{\log \log n}{\log n}\right)\right) \\
& \cdot\left(\pi \sqrt{\frac{2 \frac{6}{\pi^{2}} \cdot \log ^{2} n \cdot\left(1+O\left(\frac{\log \log n}{\log n}\right)\right)}{3}}-2 \log \left(\frac{6}{\pi^{2}} \cdot \log ^{2} n \cdot\left(1+O\left(\frac{\log \log n}{\log n}\right)\right)\right)\right)
\end{aligned}
$$

After rearranging the terms we arrive at

$$
\begin{aligned}
\log f_{3}(n) \geqslant & -\frac{\pi^{2}}{6} \cdot \frac{n}{\log ^{2} n} \cdot\left(1+O\left(\frac{\log \log n}{\log n}\right)\right) \cdot(\log n+O(\log \log n)) \\
& +\frac{\pi^{2}}{3} \cdot \frac{n}{\log ^{2} n} \cdot\left(1+O\left(\frac{\log \log n}{\log n}\right)\right) \cdot \log n \cdot\left(1+O\left(\frac{\log \log n}{\log n}\right)\right) \\
& +\frac{\pi^{2}}{6} \cdot \frac{n}{\log ^{2} n} \cdot O(\log \log n)=\frac{\pi^{2}}{6} \cdot \frac{n}{\log n} \cdot\left(1+O\left(\frac{\log \log n}{\log n}\right)\right)
\end{aligned}
$$


We proceed by induction to show the lower bound for $f_{k}(n)$ for $k>3$. Hence, assume that the estimation $\log f_{k}(n) \geqslant \frac{\pi^{2}}{6} \cdot \frac{n}{L_{k-2}(n)} \cdot\left(1+O_{k}\left(\frac{L_{k-1}(n)}{L_{k-2}(n)}\right)\right)$ holds for some $k>3$. To obtain a similar lower bound for $f_{k+1}(n)$ we use the recurrence formula (F1), that is, $F_{k+1}(x)=x \exp \left(\sum_{m=1}^{\infty} \frac{1}{m} \sum_{j=1}^{\infty} f_{k}(j) x^{j m}\right)$, which yields the estimation

$$
F_{k+1}(x) \geqslant_{\text {coeff }} x \exp \left(\sum_{n=1}^{\infty} f_{k}(n) x^{n}\right)
$$

According to the induction hypothesis there exist $n_{k} \in \mathbb{N}$ and $R_{k} \in \mathbb{R}$ such that $f_{k}(n) \geqslant \exp \left(\frac{\pi^{2}}{6} \cdot \frac{n}{L_{k-2}(n)} \cdot\left(1+R_{k} \frac{L_{k-1}(n)}{L_{k-2}(n)}\right)\right)$ for $n \geqslant n_{k}$. As $f_{k}(n) \geqslant 0$ we may omit the first few terms of the sum.

$$
F_{k+1}(x) \geqslant_{\text {coeff }} x \exp \left(\sum_{n=n_{k}}^{\infty} \exp \left(\frac{\pi^{2}}{6} \cdot \frac{n}{L_{k-2}(n)} \cdot\left(1+R_{k} \frac{L_{k-1}(n)}{L_{k-2}(n)}\right)\right) x^{n}\right)
$$

By expanding the power series of exp we obtain that for $n \geqslant 1$

$$
f_{k+1}(n+1) \geqslant \sum_{i=1}^{n} \frac{1}{i !} \prod_{n_{k} \leqslant a_{1}, \ldots, a_{i} ; a_{1}+\cdots+a_{i}=n} \prod_{j=1}^{i} \exp \left(\frac{\pi^{2}}{6} \cdot \frac{a_{j}}{L_{k-2}\left(a_{j}\right)} \cdot\left(1+R_{k} \frac{L_{k-1}\left(a_{j}\right)}{L_{k-2}\left(a_{j}\right)}\right)\right)
$$

For large enough $n$ and $x_{0}=\frac{\pi^{2}}{6} \cdot \frac{n}{L_{1}(n) \cdots L_{k-2}(n) L_{k-1}^{2}(n)}\left(1+O\left(\frac{L_{k}(n)}{L_{k-1}(n)}\right)\right)$ we have $x_{0} \geqslant n_{k}$. By setting $\log i ! \leqslant i \log i$, with $i=x_{0}$ and $a_{1}=\cdots=a_{i}=\frac{n}{x_{0}}$ we obtain

$$
\begin{aligned}
\log f_{k+1}(n+1) & \geqslant-x_{0} \log x_{0}+x_{0} \frac{\pi^{2}}{6} \cdot \frac{\frac{n}{x_{0}}}{L_{k-2}\left(\frac{n}{x_{0}}\right)} \cdot\left(1+R_{k} \frac{L_{k-1}\left(\frac{n}{x_{0}}\right)}{L_{k-2}\left(\frac{n}{x_{0}}\right)}\right) \\
& =-x_{0} \log x_{0}+\frac{\pi^{2}}{6} \cdot \frac{n}{L_{k-2}\left(\frac{n}{x_{0}}\right)} \cdot\left(1+R_{k} \frac{L_{k-1}\left(\frac{n}{x_{0}}\right)}{L_{k-2}\left(\frac{n}{x_{0}}\right)}\right)
\end{aligned}
$$

From the definition of $x_{0}$ we have

$$
\frac{n}{x_{0}}=\frac{6}{\pi^{2}} L_{1}(n) \cdots L_{k-2}(n) L_{k-1}^{2}(n) \cdot\left(1+O\left(\frac{L_{k}(n)}{L_{k-1}(n)}\right)\right)
$$

By Lemma 3.2 it follows that $L_{m}\left(\frac{n}{x_{0}}\right)=L_{m+1}(n)+O\left(L_{m+2}(n)\right)$. Finally, the estimation 
$x_{0} \log x_{0}=O\left(\frac{n}{L_{k-1}^{2}(n)}\right)$ yields

$$
\begin{aligned}
\log f_{k+1}(n+1) & \\
& \geqslant O\left(\frac{n}{L_{k-1}^{2}(n)}\right)+\frac{\pi^{2}}{6} \cdot \frac{n}{L_{k-1}(n)+O\left(L_{k}(n)\right)} \cdot\left(1+R_{k} \frac{L_{k}(n)+O\left(L_{k+1}(n)\right)}{L_{k-1}(n)+O\left(L_{k}(n)\right)}\right) \\
& =O\left(\frac{n}{L_{k-1}^{2}(n)}\right)+\frac{\pi^{2}}{6} \cdot \frac{n}{L_{k-1}(n)} \cdot\left(1+O\left(\frac{L_{k}(n)}{L_{k-1}(n)}\right)\right) \cdot\left(1+R_{k} \frac{L_{k}(n)}{L_{k-1}(n)} O(1)\right) \\
& =\frac{\pi^{2}}{6} \cdot \frac{n}{L_{k-1}(n)} \cdot\left(1+O\left(\frac{L_{k}(n)}{L_{k-1}(n)}\right)\right)
\end{aligned}
$$

Thus we arrive at the lower bound

$$
\begin{aligned}
\log f_{k+1}(n) & \geqslant \frac{\pi^{2}}{6} \cdot \frac{n-1}{L_{k-1}(n-1)} \cdot\left(1+O\left(\frac{L_{k}(n-1)}{L_{k-1}(n-1)}\right)\right) \\
& \geqslant \frac{\pi^{2}}{6} \cdot \frac{n}{L_{k-1}(n)} \cdot\left(1-\frac{1}{n}\right) \cdot\left(1+O\left(\frac{L_{k}(n)}{L_{k-1}(n)}\right)\right) \\
& =\frac{\pi^{2}}{6} \cdot \frac{n}{L_{k-1}(n)} \cdot\left(1+O\left(\frac{L_{k}(n)}{L_{k-1}(n)}\right)\right)
\end{aligned}
$$

\subsection{The upper estimation}

Lemma 4.3. We have for real $x \rightarrow 1-$

$$
\log F_{2}(x)=\frac{\pi^{2}}{6(1-x)}+\frac{1}{2} \log (1-x)-\frac{\pi^{2}}{12}-\log \sqrt{2 \pi}+O(1-x)
$$

Proof. This is a reformulation of formula (68) on p. 576 from [4]. We just note that $F_{2}(x)=x P(x)$ and that the factor $x$ leads to an (additional) error term of the form $\log x=O(1-x)$.

The next step is to extend Lemma 4.3 in a proper way for $F_{k}(x), k>2$.

Lemma 4.4. For every $k \geqslant 2$ there exists $C_{k}>0$ and $x_{0}(k)<1$ such that

$$
L_{k-1}\left(F_{k}(x)\right) \leqslant \frac{\pi^{2}}{6(1-x)}+\frac{1}{2} \log (1-x)+C_{k}
$$

for $x_{0}(k) \leqslant x<1$.

Proof. The statement is shown by induction. However, we first observe that the sum $\sum_{m \geqslant 1} F_{k}\left(x^{m}\right) / m$ can be replaced by a much simpler upper bound. For $0<x<1$ we set $m_{0}=m_{0}(x)=\lceil 1 / \log (1 / x)\rceil$. If $x_{1}<1$ is sufficiently close to 1 , then we can apply the 
estimation $F_{k}(x)=O(x)$ to obtain

$$
\begin{aligned}
\sum_{m>m_{0}} \frac{1}{m} F_{k}\left(x^{m}\right) & =O\left(\sum_{m>m_{0}} \frac{1}{m} x^{m}\right)=O\left(\sum_{m=0}^{\infty} \frac{1}{m} x^{m}-\sum_{m=0}^{m_{0}} \frac{1}{m} x^{m}\right) \\
& =O\left(-\log (1-x)-\log \left(m_{0}\right)+O(1)\right) \\
& =O\left(-\log \frac{1-x}{\log x}+O(1)\right)=O(1)
\end{aligned}
$$

which is negligible, as there will be a larger error term. Furthermore, we have

$$
\sum_{m=3}^{m_{0}} \frac{1}{m} F_{k}\left(x^{m}\right)=O\left(\frac{1}{\log (1 / x)} F_{k}\left(x^{3}\right)\right)=O\left(\frac{1}{1-x} F_{k}\left(x^{3}\right)\right)
$$

which leads to the upper bound

$$
\sum_{m \geqslant 1} \frac{1}{m} F_{k}\left(x^{m}\right)=F_{k}(x)+\frac{1}{2} F_{k}\left(x^{2}\right)+O\left(\frac{1}{1-x} F_{k}\left(x^{3}\right)\right)
$$

Finally, we prove (2) by induction. By Lemma 4.3 it is certainly true for $k=2$. So we assume now that it is true for some $k \geqslant 2$. For notational convenience we set

$$
G(x)=\frac{\pi^{2}}{6(1-x)}+\frac{1}{2} \log (1-x) .
$$

It is immediate that

$$
G\left(x^{2}\right)=\frac{\pi^{2}}{6\left(1-x^{2}\right)}+\frac{1}{2} \log \left(1-x^{2}\right)=\frac{\pi^{2}}{12(1-x)}+\frac{1}{2} \log (1-x)+O(1)
$$

as $x \rightarrow 1-$; and a similar estimation follows if we replace $x^{2}$ by $x^{3}$ :

$$
G\left(x^{3}\right)=\frac{\pi^{2}}{6\left(1-x^{3}\right)}+\frac{1}{2} \log \left(1-x^{3}\right)=\frac{\pi^{2}}{18(1-x)}+\frac{1}{2} \log (1-x)+O(1) .
$$

Since $\log (1-x) \rightarrow-\infty($ as $x \rightarrow 1-)$ we have that for every $C>0$ there exists $x_{2}=$ $x_{2}(C)<1$ such that

$$
G\left(x^{2}\right)+C \leqslant \frac{1}{2} G(x) \text { and } \quad G\left(x^{3}\right)+C \leqslant \frac{1}{3} G(x)
$$

for $x_{2} \leqslant x<1$. According to the induction hypothesis we have $F_{k}(x) \leqslant E_{k-1}\left(G(x)+C_{k}\right)$. Thus Lemma 3.2 items (iii) and (iv) imply

$$
F_{k}\left(x^{2}\right) \leqslant E_{k-1}\left(G\left(x^{2}\right)+C_{k}\right) \leqslant E_{k-1}(G(x) / 2) \leqslant E_{k-1}(G(x))^{1 / 2}
$$

and similarly

$$
\frac{1}{1-x} F_{k}\left(x^{3}\right) \leqslant \frac{1}{1-x} E_{k-1}(G(x))^{1 / 3} \leqslant E_{k-1}(G(x))^{1 / 2}
$$


provided that $x<1$ is sufficiently close to 1 . Hence, we obtain

$$
\begin{aligned}
\log F_{k+1}(x) & \leqslant \sum_{m \geqslant 1} \frac{1}{m} F_{k}\left(x^{m}\right) \\
& =F_{k}(x)+\frac{1}{2} F_{k}\left(x^{2}\right)+O\left(\frac{1}{1-x} F_{k}\left(x^{3}\right)\right) \\
& \leqslant E_{k-1}\left(G(x)+C_{k}\right)+O\left(E_{k-1}(G(x))^{1 / 2}\right) \\
& \leqslant E_{k-1}\left(G(x)+C_{k}\right)\left(1+O\left(E_{k-1}\left(G(x)+C_{k}\right)^{-1 / 2}\right)\right) \\
& \leqslant E_{k-1}\left(G(x)+C_{k+1}\right) .
\end{aligned}
$$

which is equivalent to (2) for $k+1$.

Corollary 4.5. For every $k \geqslant 2$ there exists $x_{1}(k)<1$ such that

$$
L_{k-1}\left(F_{k}(x)\right) \leqslant \frac{\pi^{2}}{6 \log (1 / x)}
$$

for $x_{1}(k) \leqslant x<1$.

Proof. Since

$$
\frac{\pi^{2}}{6(1-x)}=\frac{\pi^{2}}{6 \log (1 / x)}+O(1)
$$

and $\log (1-x) \rightarrow-\infty$ (as $x \rightarrow 1-)$, it immediately follows that (3) holds for $x_{1}(k) \leqslant x<1$, if $x_{1}(k)<1$ is large enough.

We finish the proof of the main result by verifying the upper bound.

Theorem 4.6. For every $k \geqslant 3$ we have for $n \rightarrow \infty$

$$
\left[x^{n}\right] F_{k}(x) \leqslant \frac{\pi^{2}}{6} \cdot \frac{n}{L_{k-2}(n)}\left(1+O\left(\frac{L_{2}(n)}{\log n L_{2}(n) \cdots L_{k-3}(n) L_{k-2}(n)}\right)\right) .
$$

Proof. We use the trivial inequality $f_{k}(n) x^{n} \leqslant F_{k}(x)$ (for $0 \leqslant x<1$ ) to obtain an upper bound for $f_{k}(n)=\left[x^{n}\right] F_{k}(x)$. To this end, $x$ has to be chosen in a proper way, namely by the relation

$$
\log (1 / x)=\frac{\pi^{2}}{\left.6 L_{k-2}\left(n /(\log n)^{2}\right)\right)}
$$

With this value we have by $(3)$ the inequality $L_{k-1}\left(F_{k}(x)\right) \leqslant L_{k-2}\left(n /(\log n)^{2}\right)$, and consequently $\log F_{k}(x) \leqslant n /(\log n)^{2}$. Furthermore, since

$$
\frac{\pi^{2}}{6 L_{k-2}\left(n /(\log n)^{2}\right)}=\frac{\pi^{2}}{6 L_{k-2}(n)}\left(1+O\left(\frac{L_{2}(n)}{\log n L_{2}(n) \cdots L_{k-2}(n)}\right)\right)
$$


we obtain the estimation

$$
\begin{aligned}
\log f_{k}(n) & \leqslant \log F_{k}(x)+n \log (1 / x) \\
& \leqslant \frac{n}{(\log n)^{2}}+\frac{\pi^{2}}{6} \cdot \frac{n}{L_{k-2}\left(n /(\log n)^{2}\right)} \\
& =\frac{\pi^{2}}{6} \cdot \frac{n}{L_{k-2}(n)}\left(1+O\left(\frac{L_{2}(n)}{\log n L_{2}(n) \cdots L_{k-3}(n) L_{k-2}(n)}\right)\right)
\end{aligned}
$$

which completes the proof.

\section{Acknowledgements}

The authors are grateful to the referee, who has significantly shortened the paper by showing the techniques applied for the upper estimation. The research was supproted ny OTKA Grant 109085 and P.P. Pach was partially supported by the grant TAMOP -4.2.2.B-10/1-2010-0009.

\section{References}

[1] N. Broutin and P. Flajolet. The height of random binary unlabelled trees. Discrete Math. Theor. Comput. Sci.Proc., AI, 121-134, 2008.

[2] M. Drmota Random trees. An Interplay between Combinatorics and Probability. SpringerWienNewYork, 2009.

[3] M. Drmota and B. Gittenberger. The shape of unlabeled rooted random trees. Europ. J. Combinat., 3:2028-2063, 2010.

[4] P. Flajolet and R. Sedgewick Analytic Combinatorics. Cambridge University Press, Cambridge, 2009.

[5] R. Otter. The number of trees. Ann. of Math., 49:583-599, 1948.

[6] H. Rademacher. On the expansion of the partition function in a series. Ann. of Math., 44:416-422, 1943. 